\title{
Study on the Trust Model for Knowledge Transfer
}

\author{
Lubang Wang ${ }^{1}$ and Haidong Zhong, ${ }^{2 *}$ \\ ${ }^{1,2}$ Institute of Modern Logistics, Zhejiang Wanli University, Ningbo, Zhejiang, \\ P.R.China \\ ${ }^{1} 154117176 @ q q . c o m,{ }^{2}$ zhd_1981@163.com \\ *Correspondent author
}

\begin{abstract}
Knowledge transfer $(K T)$ in organizations (including the profitable enterprises and nonprofit institutions) has a great impact on their development and improvement. The fundamental form of organizational structure can be depict by trust relationship model, which qualitatively describes the causal relationship that exists between the members in the organizations. Although quantitative investigations are restricted by the amount of sample members and their access methods, they do explain the link between trust measurement and KT to some extent. Our research shows that three types of trust, Trust-Based Command Group (CT), Trust-Based Task Group (TT) and Trust-Based Interest Group (IT), are positively correlated and KT intention will ultimately affect transfer behaviour. The empirical quantitative analysis, using the questionnaire method, indicates that the influence degree order (from strong to weak) of the three types of trust on KT is: CT, IT, TT.
\end{abstract}

Keywords: Trust model; Knowledge transfer; Organizational structure

\section{Introduction}

The importance of knowledge to organizational survival and development has become consensus among scholars. Drucker, the master of management science, even thinks the importance of knowledge is superior to that of talent, capital, land and other resources, and states that it is not just a resource. [1] There are a variety of sources from which to get knowledge for an organization, such as knowledge from other organizations in the same development environment and knowledge innovation from members in the organization. Intra-organization knowledge management has great significance in promoting intraorganization knowledge popularization, optimizing knowledge structure, improving knowledge utilization efficiency and ultimately improving organizational efficiency. Therefore, it is necessary and meaningful to study KT in organizations.

Efficient in-organization KT can be obtained through institutional guarantee or incentive mechanisms. But the orientation and effect of KT can be greatly influenced by the emotion and correlation degree on both sides, because KT will not happen under the circumstance of economic incentives or institutional arrangements within organizations. In a real KT process, it usually lean to a receiver among many knowledge expectants with basically the same qualification. KT can occur even in the condition that the economic interests of the knowledge passer are not protected. So, profound analysis of these issues from the viewpoint of trust is necessary. 


\section{Trust and KT}

\subsection{Trust Factors in $\mathrm{KT}$}

The speed and degree of KT have a close relationship with KT channel characteristics, inherent traits of knowledge and the connection degree of KT on both sides. The multidimensional nature of the transfer channels for KT provides more possibilities in terms of space and time for KT processes in the Internet age. Generally, the greater the degree of implicit knowledge, the slower the transmission speed and the narrower the transmission range. In addition, the degree of specialism in the knowledge will greatly impact its transmission speed and degree: the higher the professionalism of knowledge, the lower the spread speed, the less it is appreciated by people and the lower the transmission degree. As well as inherent traits of knowledge and its transmission channels, the correlation degree of both sides in knowledge transmission also has an important influence on KT. Organizational relationships, interaction degree and the interest relationship between both sides in terms of the knowledge will affect transmission willingness and degree. The affection includes not only the transmission willingness and content of those passing the information, but also the orientation and learning initiative of the receivers.

It is difficult to describe the correlation degree between the passers and receivers in the process of KT. Scholars find the degree of trust between passers and receivers can be used as a good index to describe their correlation degree. Huemer thinks trust can explain why and how organizational knowledge can be developed in an organization, [2] and a knowledge platform can be established based on trust between partners. [3] Portillo-Rodríguez Javier and Vizcaíno Aurora deem trust an important factor for knowledge between community members and have investigated knowledge interaction behaviour in knowledge management systems by simulation. [5] A trust network which spreads structural knowledge can be formed from emotional bonds between members in the organization. [6] Hence, an organization with little trust between members will eventually lead to serious barriers in organizational KT [7].

Organizational members exist in naturally built social networks, and trust is the basic premise of knowledge dissemination and application among them. [8] The social effect of knowledge within the organization will be affected when there is a lack of motivation or organizational environment, weak internal links or a lack of trust between members. If low trust has been formed in the organization this will influence organizational knowledge creation and sharing, and thus affect the growth of the organization. [9] Knowledge exchange speeds up the process of knowledge creation and trust between members can reduce concerns about knowledge exchange risks, making the organizational climate more conducive to the creation and transmission of knowledge. [10] All these show there are significant connections between trust and the KT process. In an organization with a lack of trust, KT will be relatively slow; the organization will develop very slowly and may even be eliminated in the era of the knowledge economy.

\subsection{Feedback Probability-Based Analysis of Trust}

To some extent trust affects KT persons, the transmission rate and transmission speed in an organization. As analysed in Section 2, quantitative characterization of trust is a difficult task, and a five level classified questionnaire is designed in empirical analysis to determine the individual's trust quantitatively. The role of trust metrics for KT can be analysed from the perspective of knowledge feedback, and feedback probability of KT can be used as a trust measurement index, which is based on the approach of mutual KT between passers and receivers. 
In the KT process, the knowledge owner will loss his (or her) "wealth", resulting in "Ricardo" rent reduction of knowledge as a social resources when exclusive rights of knowledge are lost or some degree of reduction accrues. Therefore, knowledge is usually treated as a kind of wealth. The utility functions in economics can be used to analyse the process of KT and the effect of trust degree on knowledge can be measured by feedback probability.

An organizational system can be formally defined as an $\mathrm{N}$-tuple $\mathrm{X}=\left(X_{1}, X_{2}, \ldots, X_{N}\right)$, where $\mathrm{N}$ denotes there are $\mathrm{N}$ members in the system and the Cobb-Douglas function of any staff in the system can be represented as:

$$
U_{i}=K_{i 1}^{X_{1 i}} K_{i 2}^{X_{2 i}} \ldots K_{i m}^{X_{m i}},\left(x_{j m} \geq 0, j=1,2, \ldots, m, \sum_{i=1}^{N} x_{m i}=1\right),
$$

Where $U_{i}$ denotes the utility produced different types of knowledge of any member $i, K_{i m}$ denotes the "Ricardo" rent member $i$ should pay to master knowledge $m$, and $x_{m i}$ denotes the proportion of knowledge $m$ that member $i$ masters and the total knowledge grasped by the staff.

Consider the situation of knowledge exchange of two kinds, $j$ and $k$. Member $i$ is included in one of the kinds and needs to exchange his (or her) knowledge with member $k$ to get knowledge $j$. Assume that member $i$ wants to get knowledge $j$ from another member by transferring knowledge $k$, the probability of delivering knowledge is $100 \%$ and the feedback probability of accepting one kind of knowledge from another is $p$. Obviously, the value of $p$ has a positive correlation to the trust degree between members of an organization, so $p$ can be treated as a trust indicator of the system. Feedback probability varies in each KT process, and this paper focuses only on the onetime KT process of member $i$. The proportion of member $i$ 's own knowledge $j$ to the knowledge of all members can be represented as:

$$
\begin{aligned}
& x_{j i} \text {, the knowledge is successful ly fed back to member } i \text {; } \\
& 0 \text {, knowledge } j \text { cannot feed back to member } i \text {; Successful feedback }
\end{aligned}
$$
probability of knowledge $K_{i j}$ is $p$, so the utility function of knowledge of member $i$ can be represented as:

$$
U_{i}=K_{i k}^{X_{k i}} K_{i j}^{p X_{j i}}
$$

Increase of knowledge $k$ will cause a relative decrease of "Ricardo" rent, that is $\frac{d_{U_{i}}}{d_{K_{i k}}}<0$. It can be deduced from formulation (1) that

$$
\begin{aligned}
& \frac{d U_{i}}{d K_{i k}}=\frac{x_{k i}}{K_{i k}} * U_{i}+p * \frac{x_{j i}}{K_{i j}} * U_{i} * \frac{d K_{i j}}{d K_{i k}}<0 \quad \text { (2). This can be simplified as } \\
& -\frac{d K_{i k} x_{k i}}{K_{i k}}<p * \frac{d K_{i j} x_{j i}}{K_{i j}}
\end{aligned}
$$

Apparently formula (3) is true and the left side of the formula represents the loss of the "Ricardo" rent that might be caused by transfer knowledge $K_{i k}$, while the right side of the 
formula is "Ricardo" rent after transfer knowledge $K_{i k}$. For any member $i$, the knowledge process will happen only in the case that $p * \frac{d K_{i j} x_{j i}}{K_{i j}}$ is greater than $\frac{d K_{i k} x_{k i}}{K_{i k}}$. It can be seen that the coefficient $p$ is a key factor for KT intention, because it will not change with the increase or decrease of the usefulness of knowledge. Nahapiet \& Ghoshal have pointed out that people in societies with a high degree of trust will generally be more willing to participate in social exchanges, especially cooperative exchange. [11] It can be perceived that the greater the degree of trust, the stronger the willingness to transfer knowledge and raise organizational knowledge. Conversely, a system, lack of trust structures, could lead to major KT blockages and seriously impact the performance of knowledge interactivity throughout the organization.

\section{A Trust Model Based on Organizational Structure}

\subsection{Multidimensional Characterization Analysis of Trust}

Trust degree plays an important role in the extent and speed of the KT process, and transfer willingness is a prerequisite of them. The issue is a problem-rich topic and much attention has been paid to this field. Lewicki \& Bunker divided the developmental pattern of trust into three types: Calculus-Based, Knowledge-Based and Identification-Based. In their study, CalculusBased trust and Knowledge-Based trust are both built on the calculation of economic benefits, while Identification-Based trust is constructed based on a common vision and interests among all the members. [12] Zhang P. proposed a MAX trust model and thought KT can be achieved through three trust factors: knowledge value, members' intellectual ability and the secondary benefit between them. In his opinion, the three trust factors are market trust, trust in authority and x-based trust. [13] Market trust is market oriented, in terms of exchange activities under a market competition environment. Trust is guided by the authority that comes from the arrangements of the organizational system. X-based trust can be constructed based on the psychological contract or values of identity between members, which goes against the normal economics theory in certain cases, because the trade is neither driven by economic interests nor authoritative intervention. In the literature, [14] trust is analysed in two perspectives: "from individual to organization" and "from organization to organization". The approach tends to explain the dimensions of the trust, and can be used in describing trust in financial markets.

Generally, the existing literature tries to define the source and classification of individuals' trust from multiple angles, but it is difficult to define foundations of trust classification fundamentally. Trust is a typical organization-defining characteristic, therefore organizational structure has great significance in explaining the exchange of knowledge within the organization. The paper starts with the organization's internal structure, taking into account the trust based organizational structure, to analysis the source and dimension of trust.

\subsection{Members of the Organization Structure}

The organizational structure is the basis for maintaining and operating of an organization, which attaches great importance on departmental, administrative and work relationship between its members. Traditional organization is characterized by an administrative feature, but this has been gradually flattened with the coming of the network process era. Therefore, investigation of administrative and business relations within an organization in the context of KT has great significance. 
Definition 1 Faculty Network. In an organization, a faculty network can be formed between its faculties. Formally it can be represented as $\mathrm{G}=(\mathrm{V}, \mathrm{E})$, where $\mathrm{V}$ denotes the group of all members in the organization and $\mathrm{E}$ denotes the relationship between them. In the formula E contains many types of relationships, such as leadership and being relationship, common affiliation relationship in the same primary-level organization, temporary relations formed due to participation in the same task or relationships based on having the same hobbies or emotions.

Definition 1 Group Structure. A group structure in a faculty network $G=\left(V_{s} E\right)$ can be defined as $V_{T}=\left\{V_{\mathrm{i}} \mid V_{\mathrm{i}} \in V\right\}(i=1,2,3 \ldots, n)$, where $\forall V_{\mathrm{i}} \in V_{T} \rightarrow P\left(V_{\mathrm{i}}, A_{T}\right) \quad(i=1,2,3, \ldots, n), P\left(V_{i}, A_{T}\right)$ represents member $V_{i}$ has the semantics $A_{T}$ of the predicate $P$.

The condition $\forall V_{i} \in V_{T} \rightarrow P\left(V_{i}, A_{T}\right)(i=1,2,3, \ldots, n)$ shows that a group structure is a collection of nodes with the same predicate properties, e.g., nodes in a structure are member of a certain project. Group structure in an organization contains not only the organizational structure of the administrative arrangement, but also some temporary project staff arrangements, some interest-based organizations between individuals, and so on. The intraorganizational structure of an organization with group structure is shown in Figure 1.

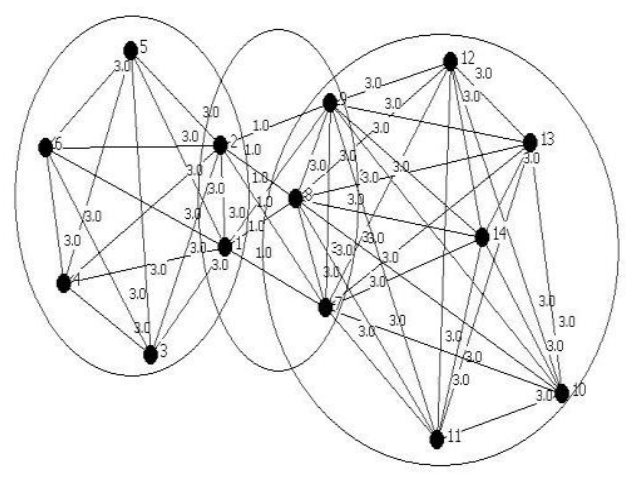

\section{Figure 1. Intra-organizational Structure of an Organization with Group Structure}

In an organizational structure, a group structure formed on the basis of a political affiliation relationship can be known as the Command Group (CG), in which the relationship between members is rather stable. The formation of a group structure formed based on temporary arrangements can be called the Task Group (TG), in which the relationship between members will end with the end of the task. In addition, the organizational structure based on the same hobbies or interests between individuals can be called the Interest Group (IG).

In an organization, $\mathrm{CG}$ is the skeleton of a structure organization and the relationship between members of the structure can generally be determined by it. In a certain CG a TG may be formed because of a task requirement or due to having the same hobbies or interests. Generally, TG will cover many different CGs and form a big task-oriented team. Meanwhile, a large IG can be formed across multiple CG and TG. It can be seen from the above analysis that there is no difference in the three kind of groups (CG, TG and IG) and crossover relationships can also exist between them. 


\subsection{Trust Dimension Based on Member Structure}

In the organizational structure, group structure, formed by intricate relationships between members, can vary greatly due to different backgrounds of the structural relationships between members. Mutual benefits are the most important motivation for information and knowledge exchange between members in a group and knowledge exchange between members of the organization is usually conducted for the sake of obtaining a return of interests or development opportunities. Staff composition affects the dissemination of knowledge in an organization and mutual trust and communication between individuals in the structural channel affects the level of knowledge and efficiency in the organization. From the point of view of membership structure in the organization, trust can be defined in the following three categories: the Trust-based Command Group (CT), the Trust-Based Task Group (TT) and the IT. IT trust is formed between members with good relationship. TT type of trust is based on the consideration of work needs and improved efficiency in accomplishing a task. CT trust is a kind of relationship formed in stable structured organizations, such as the exchange of experience between colleagues in the same department, work summary provided by the subordinate to the superior and guidance provided by the superior to a subordinate. Definition of the three types of trust is based on three kinds of organizational structures (as shown in Figure 2), and they are closely related.

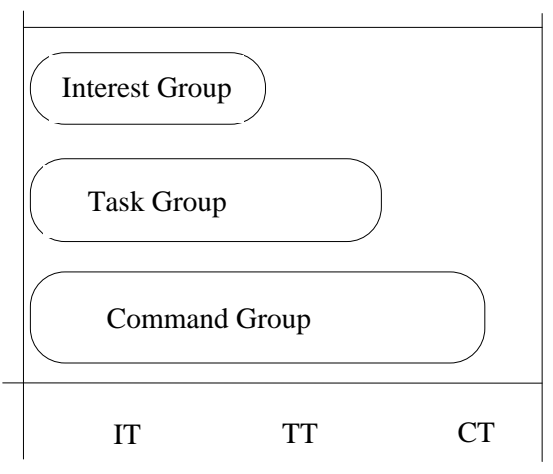

\section{Figure 2. Three Types of Structural Organization and their Correspondent Trust Categories}

IT trust, which is determined by relationships between individuals, exists in the three organizational structures within an organization. In an interest group, IT trust must exist between individuals based on the same interest. IT trust will be generated because of individuals' similar personalities or emotions in the task group and command group. TT is a kind of task-based trust relationship that generally exists in the task group and command group and CT trust only exists in the command group.

\section{Multidimensional Based Trust Analysis for KT}

\subsection{Multidimensional Trust Governance Analysis Based on Knowledge Characteristics}

Implicit characteristics which determine the difficulties involved in knowledge are the most important traits of knowledge. Deeply implicit knowledge can be treated as tacit knowledge, while comparatively easily communicated knowledge is referred to as explicit knowledge. In accordance with the intra-organization knowledge division method, Lyles \& Schwenk put forward a core-and-peripheral knowledge concept. [15] Prahalad \& Hamel 
believe that "accumulated knowledge of an organization, particularly with regard to how to coordinate the production of different skills and integrate a variety of technical knowledge of the genre" forms the core ability of the organization and provides sustainable competitive advantage. [16] Of course, external knowledge is essential for an organization, because the original core knowledge can be translated into new core knowledge in the process of combining it with the original core knowledge.

Specificity of knowledge refers to the professional knowledge, which is applied to industries with personality. Special knowledge proficient persons have very high authority in his (or her) field, but have no advantages out of this versed domain. Exclusivity of knowledge means the patent value of the knowledge, and the "exclusive" property tends to form the organizational patent value. Fang S. thinks there is no necessary link between specialized knowledge and exclusive knowledge [17]. Figure 3 shows the three-dimensional property of knowledge.

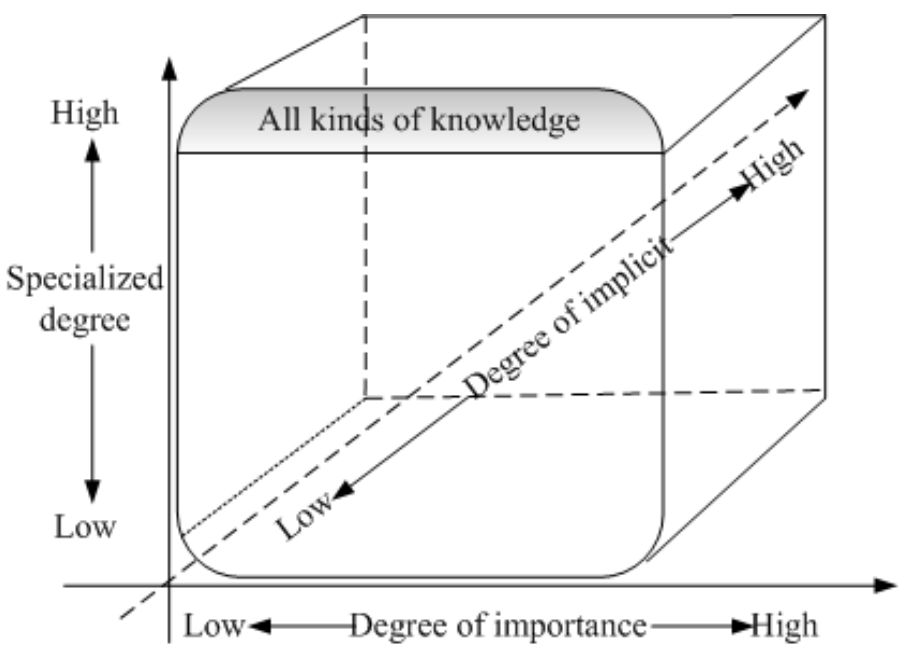

Figure 3. Three-dimensional Graph of Knowledge based on Three Properties

The three categories of trust (CT, TT and IT) in the organization have different characteristics in transmitting different kinds of knowledge. Highly important knowledge and strong specialty knowledge can be transferred through CT trust, which is based on the command structure of an organization. High specificity can be easily transferred between members by IT trust, but the transmission of highly kernel knowledge could have legal impediments because of confidentiality of the knowledge. The situation of the three types of trust transfer for three-dimensional knowledge tendency varies, as shown in Table 1, which reveals the governance scope of the three trust types. Most of the implicit knowledge transferred depends on face-to-face communication and can be achieved between IT trustbased individuals, but for those involved with the company's confidential and exclusive knowledge this is hard to accomplish. TT trust is based on the consideration of common use of individual cooperation in the team, and the most important knowledge exchange based on TT trust is dedicated knowledge, because specialized knowledge can be used to tackle the group's tasks. Additionally, highly implicit knowledge can also be transferred in a harmonious team environment, but core knowledge of the organization can hardly be transferred in a task-oriented group within a short time. CT trust fits for the transmission of a highly important and specialist knowledge, while tacit knowledge is seldom propagated in this way. 
Table 1. Three-dimension based KT Trust Dependency Status

\begin{tabular}{|l|l|l|l|}
\hline & \multicolumn{1}{|c|}{ CT } & \multicolumn{1}{c|}{ TT } & \multicolumn{1}{c|}{ IT } \\
\hline Core level & ++ & 0 & 0 \\
\hline Specialty level & + & ++ & 0 \\
\hline Implicit level & + & + & ++ \\
\hline
\end{tabular}
Notes: “++" represents "highly correlated", “+” represents “correlated”, “0” represents “little related or
irrelevant”

\subsection{Transmission Willingness of Multidimensional Trust}

In Chapter 1.2, we use thee feedback probability $p$ to represent the degree of trust between members in the knowledge exchange process. According to the multidimensional trust model, probability $p$ is the comprehensive embodiment of trust in the final delivery probability and is determined by a combination of CT trust, TT trust and IT trust from three sides with weight parameters. Formally,

$$
p=F[\|C T\|,\|T T\|,\|I T\|]
$$

There is a function to represent the relationship between knowledge and the three type of trust (CT, TT and IT). The relation between them can be quantitatively represented as:

$$
p=\alpha *\|C T\|+\beta *\|T T\|+\gamma *\|I T\|+\delta
$$

where $\|C T\|,\|T T\|$ and $\|I T\|$ are quantitative metrics of CT, TT and IT, and $\delta$ denotes a correction parameter. Figure 4 describes the relationship between the three kinds of trusts, KT willingness and KT behaviour. The three types of trust are the starting point of the problem, and also the determining factor of willingness to pass on knowledge and its delivery.

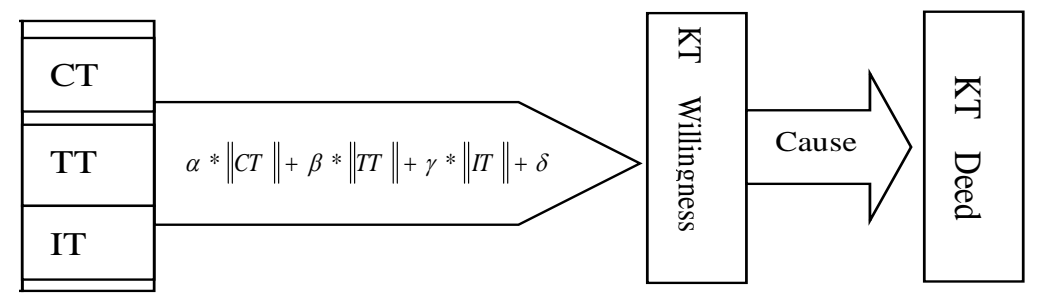

Figure 4. Relationship between Three Kind of Trusts and KT Willingness

\subsection{Study on Effect Weight of Multidimensional Trust}

To determine the weight value in the CTI model, we use questionnaire data from many enterprises from Shanghai, Shenzhen, Hangzhou and Ningbo. We delivered more than one thousand questionnaire and received about 500 valid responses. In our questionnaire the 5grade marking system $(1,2,3,4$, and 5$)$ is employed to determine the metric of trust, and the KT probability is measured by self-rating of KT from the respondent to others.

The principal component analysis and factor analysis methods are used to investigate the reasonableness of division trust into categories (CT, TT and IT). In our experiment the ball coefficient is 0.575 , which indicates the validity of the designed questionnaire for factor analysis; the Bartlett's Test value is 203.218; the DOF (degree of freedom) value is 66; and the Sig. is nearly 0, which proves the fitness of utilizing factor analysis for our questionnaire data and the category trust decomposition. Based on the result of nearly 500 questionnaires, 
we figure out the parameters value in formula (2), the value of $\alpha$ is $0.289, \beta$ is $0.106, \gamma$ is 0.231 , and $\delta$ is 0.206 . The relationship between KT willingness and three types of trust (CT, TT and IT) can be quantitatively represented as

$$
p=0.289 *\|C T\|+0.106 *\|T T\|+0.231 *\|I T\|+0.206
$$

\section{Conclusions}

The paper put forward a three-dimensional presentation model for KT and proves its reasonableness through the prospect of organizational structure and questionnaire data analysis. It is not hard to explain the positive correlation between KT probability and three types of trust, but there is a certain degree of subjectivity in the experimental data collection and the determination of quantitative models in the paper. In the future more work should be conducted to improve the accuracy of the investigation.

\section{Acknowledgements}

This work is supported by the Modern Port Service Industry and Culture Research Center of the Key Research Base of Philosophy and Social Sciences of Zhejiang Province, the Ningbo Natural Science Fundation project under Grant No. 2013A610068, the Zhejiang Province Department of Education project under Grant No. Y201329219 and the Science and Technology Innovation Team of Ningbo under Grant No. 2013B82009.

\section{References}

[1] P. F. Drucker, "Post-capitalist society", London, Butterworth Henemann, (1993).

[2] L. Huemer, G. Krogh and J. Roos, "Knowledge and the concept of trust", Newbury Park: Sage Publications, (1998).

[3] J. G. March and J. P. Olsen, "Can model of decision making in organizations", Marshefiield, MA: Pitman, (1986).

[4] J. Portillo-Rodríguez and A. Vizcaíno, "Fostering knowledge exchange in virtual communities by using agents", Proceedings of the 13th International Workshop on Groupware, Bariloche, Argentina, (2007) September 16-20.

[5] S. Juan Pablo and V. Aurora, "Applying trust, reputation and intuition aspects to support virtual communities of practice", Proceedings of the 11th International Conference on Knowledge-Based and Intelligent Information and Engineering Systems, Vietri sul Mare, Italy, (2007) September 12-14.

[6] K. Weick and K. H. Roberts, "Collective Mind in organizations: heedful interrelating on fight desks", Administrative Quarterly, vol. 38, no. 5, (1993).

[7] G. Szulanski, "Exploring internal stickiness: Impediment to the transfer of best practice within the firm", Strategic Management Journal, vol. 17, (1996).

[8] J. Scott Holste and D. Fields, "Trust and tacit knowledge sharing and use", Journal of Knowledge Management, vol. 14, (2009).

[9] D. DeLong and L. Fehey, "Diagnosing cultural barriers to knowledge management", Academy of Management Executive, vol. 14, no. 4, (2000).

[10] J. Roberts, "From know-how to show-how? Questioning the role of information and communication technologies in knowledge transfer", Technology Analysis\& Strategic Management, vol. 2, no. 4, (2000).

[11] J. Nahapiet and S. Ghoshal, "Social capital, intellectual capital, and the organizational advantage", Academy of Management Review, vol. 2, (1998).

[12] R. J. Lewick and B. B. Bunker, "Trust in relationships: a model of trust development and decline", San Francisco: Josssey-Bass, (1995).

[13] Z. Peng-cheng, "A Study on Organizational Intelligence Oriented Knowledge Transfer Mechanism and Its Effectiveness", Wuhan: Huazhong University of Science and Technology, (2005).

[14] T. Khalifa1 and A. Cesar, "A vector based model approach for defining trust in multi-organization environments", Proceedings of the 7th International Conference on Risks and Security of Internet and Systems, Cork, Ireland, (2012) October 10-12. 
[15] M. A. Lyles and C. R. Schwenk, "Top Management, Strategy, and Organizational Structures", The Journal of Management Studies, vol. 3, (1992).

[16] C. K. Prahalad and G. Hamel, "The core competencies of the corporation", Harvard Business Review, (1990), pp. 5-6.

[17] Y. Jing and K. Jie, "Research on knowledge classification and organization knowledge", Enterprise Economy, vol. 4, (2007).

\section{Authors}

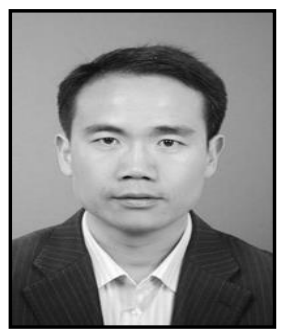

Lubang Wang, an associate professor of Modern Logistics School in Zhejiang Wanli University. He received his MCS in school of information science from SUN YAT-SEN University in 2004, and his D.E in school of management from University of Shanghai for Science and Technology in 2011. At present, his main research interest is in knowledge management, smarter city and mobile internet.

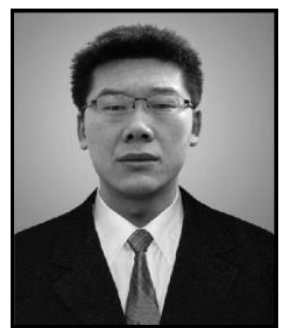

Haidong Zhong, is a lecture in the faculty of Modern Logistics School in Zhejiang Wanli University. He received his B.S in computer science and technology in 2004 and his PhD in cartography and geography information system from East China Normal University in 2011. At present, his main research interest is in mobile e-commerce, personalized recommendation and business intelligence. 